\title{
Preparation of Ex Vivo Rodent Phantoms for Developing, Testing, and Training MR Imaging of the Kidney and Other Organs
}

\author{
Jason M. Millward, João S. Periquito, Paula Ramos Delgado, \\ Christian Prinz, Thoralf Niendorf, and Sonia Waiczies
}

\begin{abstract}
Here we describe a simple and inexpensive protocol for preparing ex vivo rodent phantoms for use in MR imaging studies. The experimental animals are perfused and fixed with formaldehyde, and then wrapped with gauze and sealed with liquid latex. This yields a phantom that preserves all organs in situ, and which avoids the need to keep fixed animals and organs in containers that have dimensions very different from living animals. This is especially important for loading in MR detectors, and specifically the RF coils, they are usually used with. The phantom can be safely stored and conveniently reused, and can provide MR scientists with a realistic phantom with which to establish protocols in preparation for preclinical in vivo studies-for renal, brain, and body imaging. The phantom also serves as an ideal teaching tool, for trainees learning how to perform preclinical MRI investigations of the kidney and other target organs, while avoiding the need for handling living animals, and reducing the total number of animals required.

This protocol chapter is part of the PARENCHIMA initiative "MRI Biomarkers for CKD" (CAl6103), a community-driven Action of the European Cooperation in Science and Technology (COST) program of the European Union, which aims to improve the reproducibility and standardization of renal MRI biomarkers.
\end{abstract}

Key words Ex vivo methods, Rat, Mouse, Phantom, Magnetic resonance imaging (MRI), Kidney

1 Introduction

Prior to starting any preclinical in vivo MRI study, it is essential to invest time to test novel imaging techniques, optimize MR pulse sequence parameters and develop a workflow tailored to address the specific scientific questions at hand [1]. Much of this work can be done without using living animals, by constructing synthetic phantoms using materials that have similar MR properties as the tissue of interest (e.g., agarose). Ultimately, a point is reached when the limitations of synthetic phantoms impede progress, and phantoms that are more biologically realistic are needed. In some cases this 
can be accomplished using ex vivo tissue samples removed from experimental animals postmortem. The tissues are chemically fixed (usually with formaldehyde), and samples embedded in tubes or containers for imaging. However, this removes the organs from their surrounding environment. In the case of renal $\mathrm{MR}$, it is advantageous to image the kidney in situ, within its biological context, including the surrounding organs and perirenal fat. The typical solution for this problem is to use ex vivo phantoms, which consist of the intact experimental animal (usually rats or mice) placed inside a sealed container along with the fixation solution. This solution is suboptimal. The containers used generally have a cylindrical or rectangular shape, which is substantially different from the shape of an animal. This can be problematic given the space constraints of the MR hardware (e.g., RF coil diameter, size of the animal holding bed). In some cases the container size may mean that a larger field of view needs to be acquired than would otherwise be necessary for a living animal. It may also be difficult, or impossible, to use surface RF coils to scan samples embedded in a container. For example, when scanning the brain or the kidney, positioning the sample such that the target organ is as close as possible to the RF coil could be problematic if the container is in the way. Furthermore, the additional mass of the fixation liquid in a container with a rat or mouse body can substantially change the loading in the coil, which could cause problems with matching and tuning. The shape of the container can also cause other problems; the curved and conical shapes of typical laboratory plastic tubes can interfere with shimming the main magnetic field $B_{0}$.

Here we describe a simple and inexpensive method to prepare ex vivo phantoms using mice or rats that are perfused and chemically fixed, covered in gauze and sealed with latex. This solution results in a phantom that preserves the biological context of the organs of interest, and which is much more similar to the original animal in terms of dimensions and loading. It avoids the use of containers of toxic formaldehyde solution. The phantoms can be readily used by imaging scientists and scientists from related fields who are not trained in the handling of living experimental animals. This makes the phantom an ideal teaching tool for educating trainees learning how to perform preclinical studies, while also avoiding the confounding effects of motion artifacts. Finally, the phantoms can be easily stored and reused for extended periods of time. This will substantially reduce the total number of animals required for developing new MRI methods, and will facilitate more efficient and effective in vivo studies, thus consistent with the $3 \mathrm{R}$ principles of animal research $[2,3]$.

This chapter is part of the book Pohlmann A, Niendorf T (eds) (2020) Preclinical MRI of the Kidney-Methods and Protocols. Springer, New York. 


\section{Materials}

\subsection{Animals}

\subsection{Perfusion and Dissection}

The method described here can be used for any animal strain. The choice of strain depends on the specific needs of the researcher. Ideally, the specific rat or mouse strain should be consistent with the strain that will be used for the corresponding in vivo experiments. Most importantly the size and geometry of the animal should be comparable to that of the animal used for in vivo experiments, to ensure that the loading will be similar between phantom and in vivo experiments.

All steps of the protocol should be conducted in accordance with procedures approved by the local Animal Welfare Department and authorities and conform to guidelines to minimize discomfort to animals $(86 / 609 /$ EEC $)$.

The perfusion should be done in a lab environment equipped for dealing with hazardous fumes, using a chemical fume hood (alternatively fume extraction dome hoods) due to the respiratory hazards of formaldehyde. The lab should also have appropriate mechanisms in place for disposing of formaldehyde liquid waste, solid materials contaminated with formaldehyde (e.g., paper tissues), as well as animal carcasses (e.g., freezer for temporary storage; capacity for incineration).

1. Anesthesia (e.g., ketamine-xylazine various suppliers). Or alternative for terminal anesthesia, as per individual institutional experimental animal use guidelines.

2. Surgical instruments (scissors, rongeurs, forceps, hemostat).

3. Syringes $(1 \mathrm{ml}, 20 \mathrm{ml}, 60 \mathrm{ml})$; needles (26 Gauge, 23 Gauge Butterfly).

4. $70 \%$ ethanol in a spray bottle.

5. Solutions for perfusion: phosphate buffered saline (PBS); formaldehyde solution (e.g., 4\% aq. paraformaldehyde [PFA]).

6. Airtight plastic container for storage of the animal during the post-fixation step ( see Note $\mathbf{1}$ ).

\subsection{Latex Covering}

1. Liquid latex (e.g., 0.3\% ammonia prevulcanized, low-viscosity-various suppliers).

2. Surgical gauze.

3. 3-O surgical suture (e.g., Ethicon.com, product code C003D).

4. Fine paintbrushes.

5. Hair dryer. 


\section{Methods}

\subsection{Perfusion and Fixation}

1. Administer a terminal dose of anesthetic-here we used an intraperitoneal injection of a mixture of ketamine (100 mg/ $\mathrm{kg}$ ) and xylazine $(5 \mathrm{mg} / \mathrm{kg}$ ) diluted in $0.9 \% \mathrm{NaCl}$. We used the same dose for mouse and rat, adjusted for individual body weight.

2. Confirm that the animal has reached the required depth of anesthesia, for example by checking for absence of a reflex response to stimulation of the hind paws.

3. Place the animal in supine position on a work surface (e.g., Styrofoam covered with aluminum foil), and fix the extremities of the animal to the surface.

4. Apply $70 \%$ ethanol to the ventral surface, taking care not to spray the facial region.

5. With forceps, grasp the sternum, and make an incision into the thoracic cavity with large scissors.

6. Carefully expand the thoracic space with the scissors, taking care not to cut any major blood vessels. Cut down the ribs at the lateral sides to allow for blood drainage. Carefully separate the heart from the surrounding connective tissue. Cut through the diaphragm, and expose the inferior vena cava and the descending aorta. Attach a hemostat clamp to the sternum, and reflect backward to reveal the now opened thoracic cavity.

7. Visualize the separation between the left and right ventricles (slight color difference). Insert the needle into the left ventricle of the heart. Take care not to insert the needle too far, or you will enter the right ventricle. ${ }^{*}$ MOUSE-inject a total volume of $20 \mathrm{ml}$ PBS, using a $20 \mathrm{ml}$ syringe with a $26 \mathrm{G}$ needle. * RAT_inject a total volume of $180 \mathrm{ml}$ PBS, using $60 \mathrm{ml}$ syringes attached to a tube connected to a $23 \mathrm{G}$ butterfly needle. After inserting the needle into the heart, use a hemostat to clamp the butterfly needle. Cut the inferior vena cava.

8. Start PBS perfusion by slowly depressing the plunger, alternatively use a perfusion pump if available. Observe the color change in the liver: as a sign that the perfusion is correct, the deep red color changes to dull brown as the blood is drained (see Note 2) *RAT_-after finishing with the first $60 \mathrm{ml}$ syringe, replace the empty syringe with a filled one. Repeat with a third syringe to complete the full $180 \mathrm{ml}$ volume.

9. Start fixation by perfusing with $4 \%$ PFA (see Note 3 ) (this step should be done under a ventilation system, to avoid exposure to the hazardous PFA fumes). ${ }^{*}$ MOUSE - switch from the $20 \mathrm{ml}$ PBS syringe to a syringe filled with $20 \mathrm{ml}$ PFA. Reinsert the needle in the left ventricle, into the same injection point, 
used by the previous needle (look for a small dark spot). * RAT - attach the $60 \mathrm{ml}$ syringe with PFA directly to the tube connected to the butterfly needle. At this point the exsanguination and perfusion is complete.

10. Postfixation. Place the animal body into a sealable plastic container and fill with PFA and stored at room temperature for approximately 1 week (see Note $\mathbf{1}$ ).

\subsection{Disarticulation}

Removal of the extremities (disarticulation) can be performed at the discretion of the researcher, depending on the objective, and depending on the space constraints of the specific hardware (RF coil diameter, dimensions of the animal holding bed) that the animal phantom is to be used with. In some cases it may be sufficient to secure the forelimb and hind limb into position by suturing them to the body, before wrapping the body with gauze. Regardless, the tail should be removed. In addition, it may be necessary to remove the skin, in order to tailor the size of the phantom to the hardware dimensions. Here we describe in detail how to perform the disarticulation on a rat. In the mouse, this is easily accomplished using standard surgical scissors.

1. Remove the skin around the joint of the forelimb and the shoulder (scapular joint) with surgical scissors. Using a scalpel, dissect the musculature around the scapula (Fig. la). Using the fingers, feel for the location of the joint (Fig. lb). When the joint is exposed, use rongeurs to separate the scapula, and remove the entire forelimb (Fig. 1c). Repeat the process on the other side.

2. The procedure for the hind limbs is similar. Remove the skin around the joint of the femur and hip. Using a scalpel, dissect the musculature around the femur, and feel for the location of the ball and socket hip joint (where the femur fits in the acetabulum of the pelvis). When the joint is exposed, cut the tendons with a scalpel, or cut the bone directly using rongeurs (Fig. la). Repeat on the other side.

3. Use a scalpel to dissect the musculature of the tail at the proximal end. Expose the coccyx, and separate the tail using rongeurs. The disarticulation is now complete.

4. Proceed with removing the skin of the remaining body. Using scissors, cut through the skin at the level of the neck-the skin of the head can remain in place. Remove the skin and any extraneous tissue from the exposed thoracic cavity and the dorsal thoracic region. Use the fingers to separate the skin and expand the subcutaneous space in the dorsal lumbar region. Make a midline cut of the skin with scissors, and use a scalpel to separate the rest of the skin from the underlying 

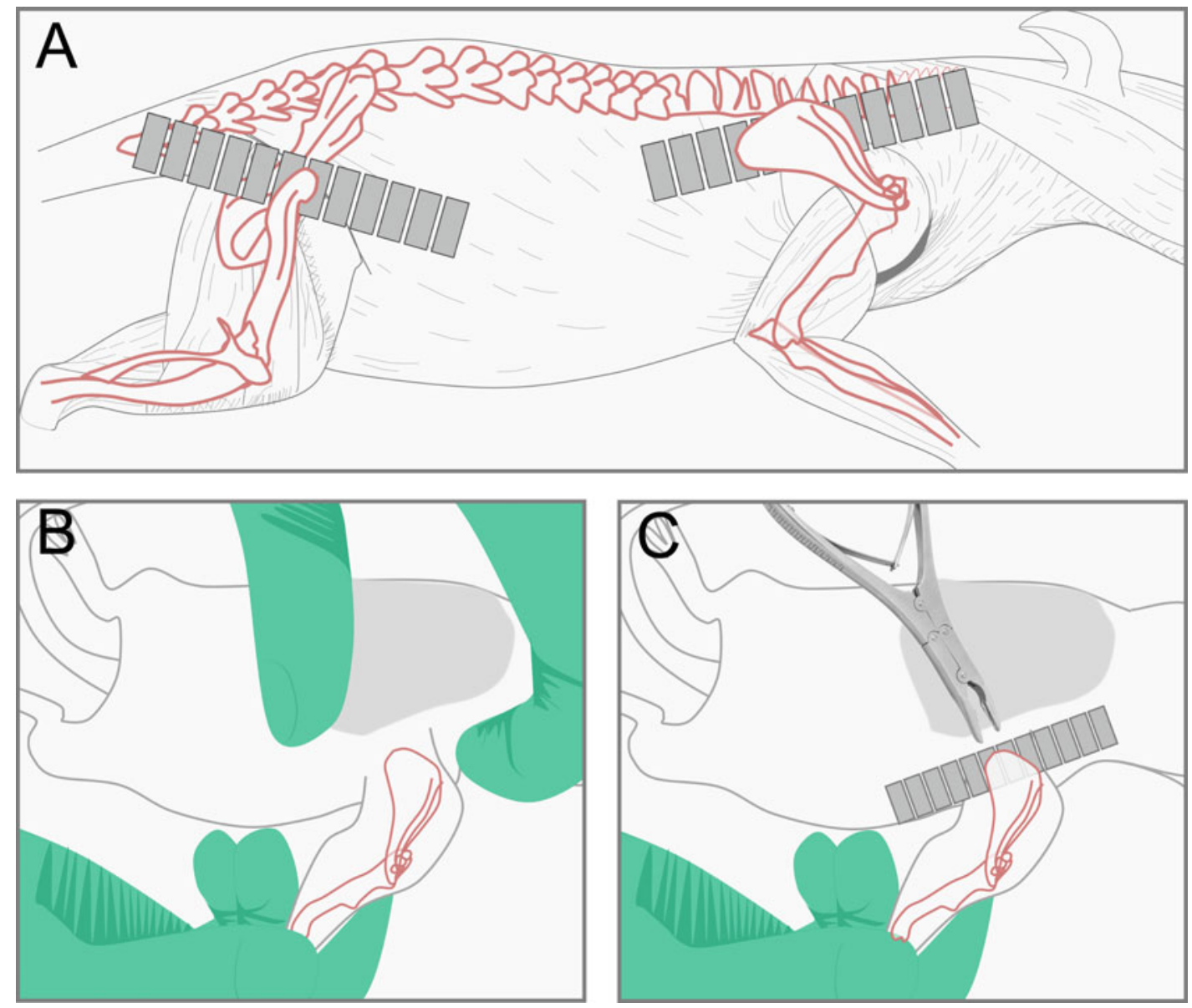

Fig. 1 Schematic for disarticulation. The location of the cut-points for removing the forelimb and hind limb is depicted by the gray lines (a). After cutting the overlying musculature, the joint of the scapula can be visualized (b). The forelimb is reflected back, and the joint cut using rongeurs (c)

tissue. Continue removing skin from the ventral lumbar region, taking care not to pierce the peritoneum.

5. The skin of the head can remain in place, since the head will not be covered with gauze. Remove the ears (exposing the auditory cavity), trim the whiskers and remove extraneous tissue from the lower mandible region.

3.3 Coating with Gauze and Latex
In the next steps the fixed and disarticulated body will be wrapped in gauze and covered in liquid latex.

1. Starting from the neck, tightly wrap the thoracic region with gauze. It may be necessary to fill part of the thoracic cavity that was opened during the perfusion step with gauze, in order to eliminate empty space and have a securely wrapped phantom. Continue wrapping the lumbar/sacral region with gauze; a 

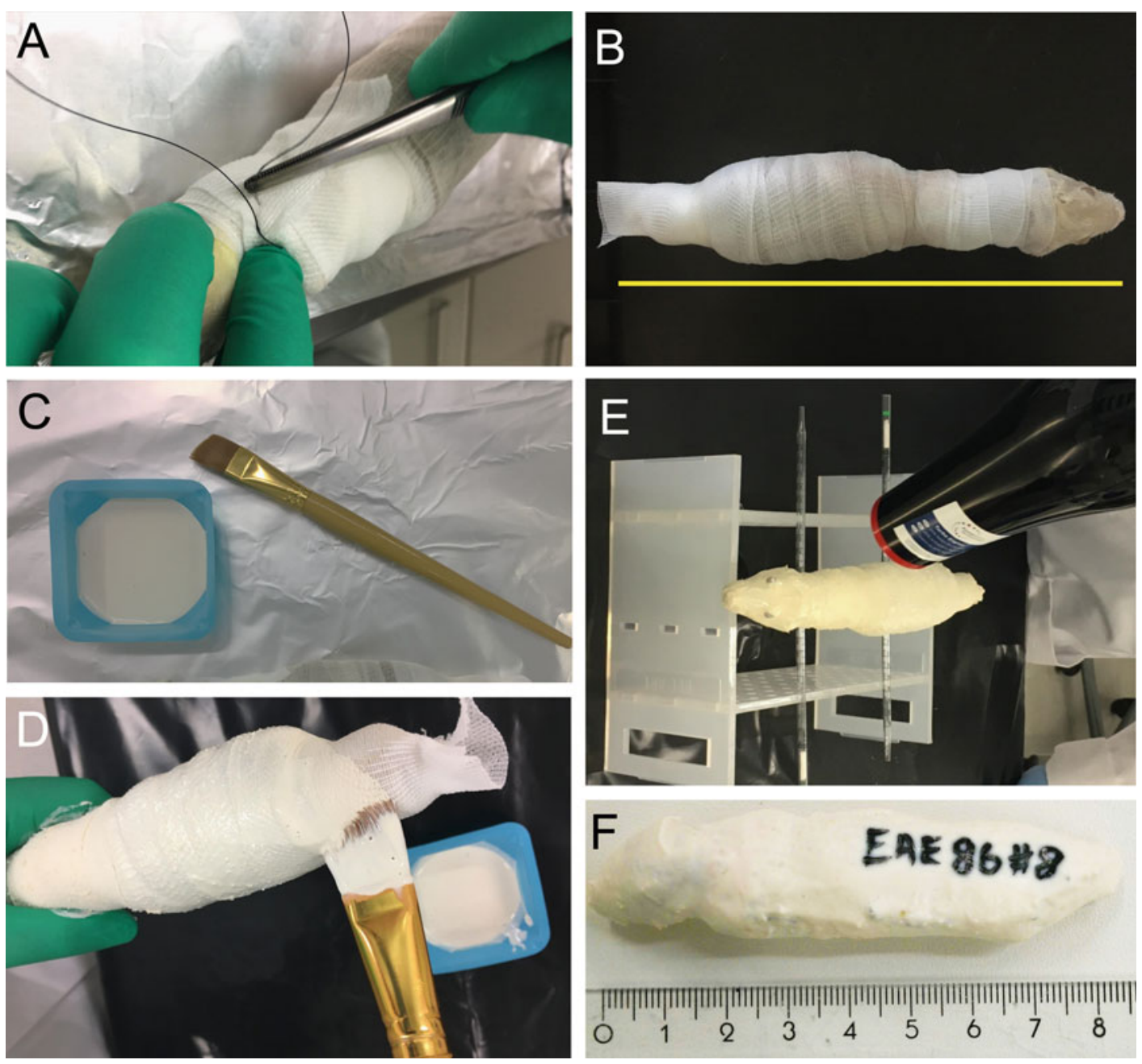

Fig. 2 Workflow for preparation of the phantom. The rat body is wrapped tightly with gauze, which is then fixed securely to the underlying musculature using surgical sutures (a). The entire body is covered with gauze, except for the head, which remains unwrapped (scale bar $=22 \mathrm{~cm})(\mathbf{b})$. Liquid latex is applied directly to the surface of the phantom using a paintbrush $(\mathbf{c}, \mathbf{d})$. The phantom is place on an improvised holder, here a laboratory tube rack with pipettes. A hair dryer is used to accelerate the drying process (e). An example of a completed, labeled mouse phantom (f)

small extension of gauze can remain at the posterior end to serve as a grip when applying the latex.

2. Firmly attach the gauze to the underlying muscle tissue using standard surgical sutures (Fig. 2a). The phantom is now ready to be covered with latex (Fig. 2b).

3. Pour some liquid latex into a suitable container; for example, a plastic weigh boat used with a standard laboratory scale is ideal. Using a flat paintbrush, apply the first coat of latex directly to the gauze (Fig. 2b, c). All surfaces should be completely 
covered, although avoid excessive latex. It is preferable to apply the latex with a brush, rather than simply submerging the phantom in latex.

4. Generously apply latex to the head, taking care to fill the auditory cavity and the oral cavity.

5. Place the phantom on a support to dry, allowing air to circulate. Here we improvised a support using a laboratory tube rack and pipettes (Fig. 2d).

6. Use a standard hair dryer to apply warm air (c. 5-10 min), to accelerate the drying of the latex (Fig. $2 \mathrm{~d}$ ). Allow additional time (c. $20 \mathrm{~min}$ ) to dry at room temperature.

7. The phantom will be easier to handle once the first coat of latex is dry. Then, apply a second coat of liquid latex, ensuring that all gaps are completely covered. Make sure that the oral cavity is completely sealed. Apply the latex generously; the objective is to ensure that there is a complete watertight seal over the entire surface. Repeat the drying process with the hair dryer, and allow to air dry completely at room temperature.

8. The phantom is now finished. It is possible to write on the surface of the dry latex with a permanent felt-tipped marker, for example to record useful information about the animal (Fig. 2f).

9. The phantom can be used at room temperature, for example inside the MRI scanner, for extended periods. Long-term storage should be at $4{ }^{\circ} \mathrm{C}$.

\section{Example Images}

\subsection{Rat}

In Fig. 3 we show representative images of MRI scans of the ex vivo phantoms. These scans were performed on a Bruker Biospec 9.4 Tesla USR94/20 animal MRI scanner (Bruker Biospin, Ettlingen, Germany).

MRI scans targeting the rat kidney were acquired in the coronal (Fig. 3a, b) and sagittal (Fig. 3d, e) planes. The identical MRI hardware setup (including the RF coils) and pulse sequence parameters were used to acquire the images of the ex vivo phantom (Fig. 3a, d) and an in vivo rat of the same strain and approximate mass as the animal used to prepare the phantom (Fig. 3b, e). The in vivo scans were done as described in the chapter by Pohlmann A et al. "Experimental Protocol for MRI Mapping of the Blood Oxygenation-Sensitive Parameters $\mathrm{T}_{2}{ }^{*}$ and $\mathrm{T}_{2}$ in the Kidney." The ex vivo and in vivo scans show a comparable level of detail, and the kidneys (denoted by " $\mathrm{K}$ ") can be seen in relation to the surrounding tissues. MRI sequence parameters are listed in Note 4. 
rat ex vivo phantom

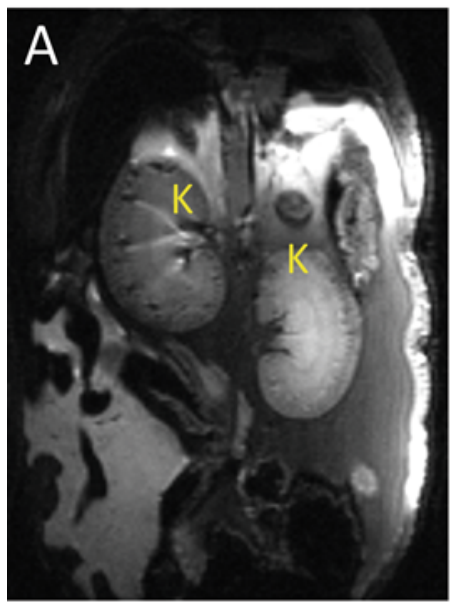

D

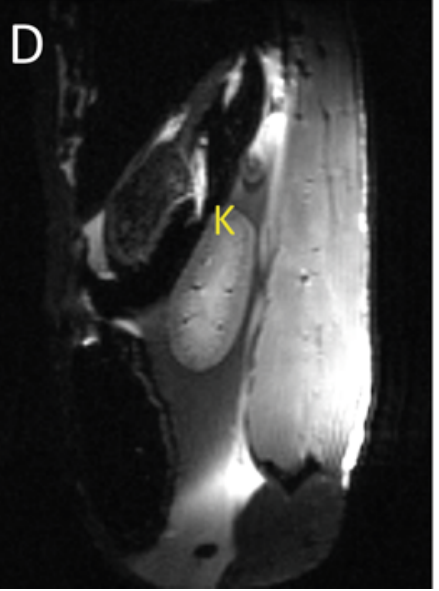

rat in vivo
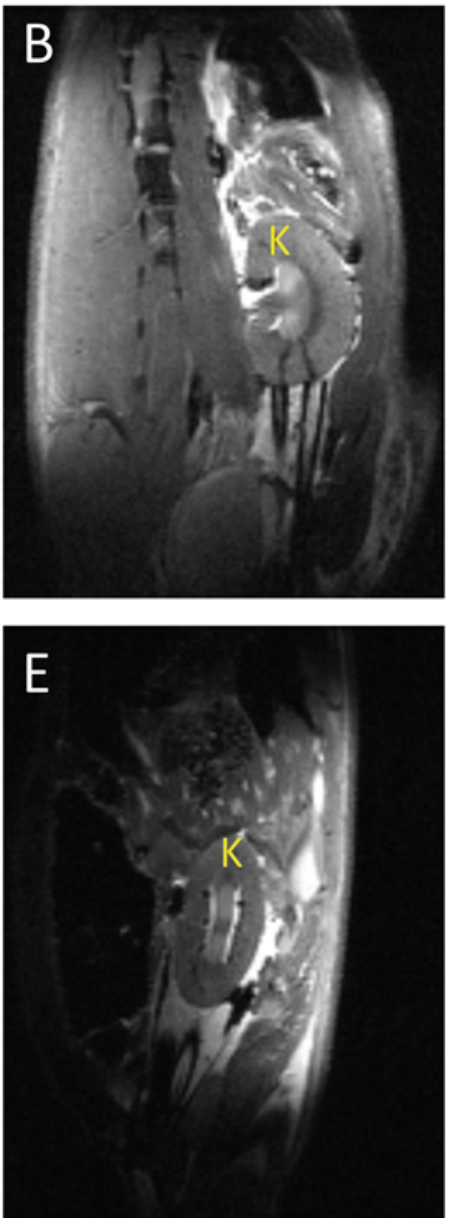

mouse ex vivo phantom
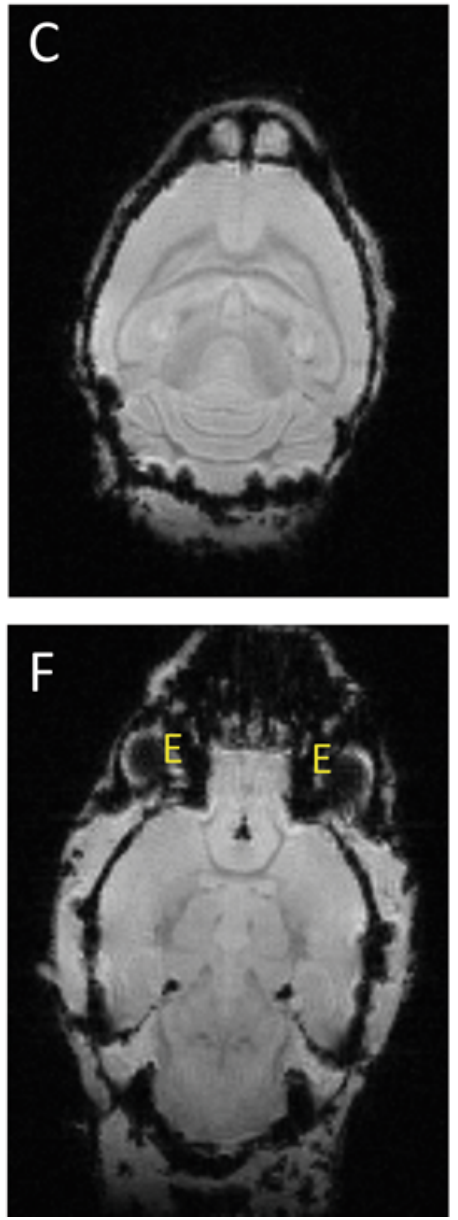

Fig. 3 Representative ex vivo and in vivo MR images. MRI scans targeting the rat kidney were acquired in the coronal $(\mathbf{a}, \mathbf{b})$ and sagittal $(\mathbf{d}, \mathbf{e})$ planes. Identical MRI hardware and pulse sequence parameters were used to acquire the images of the ex vivo phantom $(\mathbf{a}, \mathbf{d})$ and in vivo images of a rat of the same strain and approximate mass as the animal used to prepare the phantom $(\mathbf{b}, \mathbf{e})$. The kidneys are denoted by "K." MRI scans targeting the mouse brain were acquired in the coronal plane in the ex vivo phantom (c, f). Images show the brain in relation to surrounding structures such as the skull, and the eyes (denoted by "e")

\subsection{Mouse}

MRI scans targeting the mouse brain were acquired in the coronal plane in the ex vivo phantom (Fig. 3c, f). A mid-brain slice shows good contrast between gray matter and white matter structures, and the skull can be seen on the exterior of the brain (Fig. 3c). A lower brain slice (from a more inferior level) shows the brain in relation to surrounding tissue structures, for example the eyes (denoted by "E") (Fig. 3f). MRI sequence parameters are listed in Note 5. 


\section{Notes}

1. The container should be large enough that the animal is completely submerged in the formaldehyde solution and also suited for preserving the shape of the animal (e.g., curvature of the body) during postfixation.

2. Ensure that no PBS leaks from the mouth and nose region: this is a sign that the needle has shifted position into the right ventricle (and that the PBS is being perfused into the respiratory circulation, not the systemic circulation). If this occurs, remove the needle and reposition into the left ventricle.

3. Formaldehyde fixation solutions such as $4 \%$ aq. PFA or $10 \%$ neutral buffered formalin are widely used, but other fixation solutions may be substituted, as suit the needs of the experimenter. One variation is to add $8 \mathrm{mM}$ of gadolinium-based contrast agent, to change the relaxation properties of the tissue in the ex vivo preparation [4]. Another alternative is to use a zinc-based fixation solution [5].

4. MR images of the rat ex vivo and in vivo were acquired using a RARE sequence: $\mathrm{TR}=540 \mathrm{~ms}, \mathrm{TE}=12 \mathrm{~ms}$, $\mathrm{FOV}=70 \times 52 \times 35 \mathrm{~mm}$, matrix $=128 \times 172 \times 5$, number of averages $=4$, slice thickness $=0.7 \mathrm{~mm}$. acquisition time $=1 \min 9 \mathrm{~s}$.

5. MR images of the mouse ex vivo phantom were acquired using a FLASH sequence: $\mathrm{TR}=50 \mathrm{~ms}$, $\mathrm{TE}=8 \mathrm{~ms}$, $\mathrm{FOV}=20 \times 20 \times 12 \mathrm{~mm}$, matrix $=160 \times 160 \times 96$, number of averages $=4$, slice thickness $=12 \mathrm{~mm}$, acquisition time $=56 \min 32 \mathrm{~s}$.

\section{Acknowledgments}

This work was funded in part (Thoralf Niendorf, Sonia Waiczies, Joao Periquito) by the German Research Foundation (Gefoerdert durch die Deutsche Forschungsgemeinschaft (DFG), Projektnummer 394046635, SFB 1365, RENOPROTECTION. Funded by the Deutsche Forschungsgemeinschaft (DFG, German Research Foundation), Project number 394046635, SFB 1365, RENOPROTECTION).

This chapter is based upon work from COST Action PARENCHIMA, supported by European Cooperation in Science and Technology (COST). COST (www.cost.eu) is a funding agency for research and innovation networks. COST Actions help connect research initiatives across Europe and enable scientists to enrich their ideas by sharing them with their peers. This boosts their research, career, and innovation. 
PARENCHIMA (renalmri.org) is a community-driven Action in the COST program of the European Union, which unites more than 200 experts in renal MRI from 30 countries with the aim to improve the reproducibility and standardization of renal MRI biomarkers.

\section{References}

1. Cunha L, Horvath I, Ferreira S, Lemos J, Costa P, Vieira D, Veres DS, Szigeti K, Summavielle T, Mathe D, Metello LF (2014) Preclinical imaging: an essential ally in modern biosciences. Mol Diagn Ther 18(2):153-173. https://doi.org/10.1007/s40291-013-0062-3

2. Graham ML, Prescott MJ (2015) The multifactorial role of the $3 R$ s in shifting the harm-benefit analysis in animal models of disease. European J Pharmacol 759:19-29. https://doi.org/10. 1016/j.ejphar.2015.03.040

3. Russell WMS, Burch RL (1959) The principles of humane experimental technique. Methuen \& Co. Ltd., London
4. Cleary JO, Wiseman FK, Norris FC, Price AN, Choy M, Tybulewicz VL, Ordidge RJ, Brandner S, Fisher EM, Lythgoe MF (2011) Structural correlates of active-staining following magnetic resonance microscopy in the mouse brain. NeuroImage 56(3):974-983. https:// doi.org/10.1016/j.neuroimage.2011.01.082

5. Mori H, Soonsawad P, Schuetter L, Chen Q, Hubbard NE, Cardiff RD, Borowsky AD (2015) Introduction of zinc-salt fixation for effective detection of immune cell-related markers by immunohistochemistry. Toxicol Pathol 43(6):883-889. https://doi.org/10.1177/ 0192623315587593

Open Access This chapter is licensed under the terms of the Creative Commons Attribution 4.0 International License (http://creativecommons.org/licenses/by/4.0/), which permits use, sharing, adaptation, distribution and reproduction in any medium or format, as long as you give appropriate credit to the original author(s) and the source, provide a link to the Creative Commons license and indicate if changes were made.

The images or other third party material in this chapter are included in the chapter's Creative Commons license, unless indicated otherwise in a credit line to the material. If material is not included in the chapter's Creative Commons license and your intended use is not permitted by statutory regulation or exceeds the permitted use, you will need to obtain permission directly from the copyright holder. 